\title{
Les politiques actuelles permettent-elles d'atteindre un développement durable en agriculture ? *
}

Jean-Christophe KROLL

ENESAD

UMR CESAER, INRA-ENESAD

$<j$ c.kroll@enesad.fr>

\begin{abstract}
Sustainable development is a concept in course of construction which needs materialize throught precise indicators to become operating. In agriculture, this indicators are laking and it is difficult to evaluate the impact of agricultural policies on sustainable development. In the economic area, where indicators exist, the evaluation shows the rough impact of the deletion of direct subsidies on the increase in unemployment, in unexploited areas, and on cutting-down in production. This observation brings in question the sustainability of the actual agricultural policies in the long run, and brings in question the theorical postulates these policies are steming from. Beside the questions about economic sustainability, many examples show the contradictions of these policies in matter of social and environnemental sustainability. All these questions shows the long way which remain to be cover to built the new governance forms necessary to set up agricultural policies that can promote a real sustainable agriculture.
\end{abstract}

Key words: sustainable development, indicators, governance

contradictions qui subsistent dans la définition des objectifs d'une agriculture plus durable sur le plan social, et le chemin qui reste à parcourir pour avancer vers une durabilité environnementale effective.

Sans être exhaustives, les investigations partielles qui peuvent être menées tendent à montrer que les politiques actuelles ne favorisent pas l'affirmation d'un développement durable en agriculture, fondé sur des principes politiques soutenables, qui permette de dépasser l'horizon des ajustements à court terme, au coup par coup. L'une des raisons essentielles de cette impuissance trouve certainement son origine dans les formes contemporaines de gouvernance qui se mettent en place, caractérisées par le recul des Etats-Nations et l'affirmation du pouvoir d'instances internationales multiples, régionales ou planétaires, qui ne sont pas en situation politique de pouvoir réaliser les arbitrages entre la croissance économique, le développement social, et la responsabilité environnementale nécessaires à la progression vers un développement agricole plus durable.

Dans un tel contexte, compte tenu des caractéristiques tout à fait spécifiques des différentes agricultures du monde, la fuite en avant incontrôlée dans la libéralisation des échanges agricoles et alimentaires constitue une voie sans issue. Il apparaît alors nécessaire de consolider des institutions régionales reconnues et efficaces, susceptibles de réaliser les arbitrages nécessaires à un développement agricole durable. En ce domaine, l'Union européenne constitue un exemple de coordination des politiques plutôt réussi, qui pourrait jouer un rôle moteur dans la promotion d'une agriculture durable, à condition de ne pas diluer les prin- cipes fondateurs de la politique agricole commune dans une globalisation non maîtrisée des échanges.

\section{Le développement durable : un concept en construction}

Le développement de l'agriculture est aujourd'hui en question. Dans les pays riches et notamment dans I'Union européenne, les modèles techniques de production qualifiés de « productivistes », qui ont permis l'augmentation rapide de la productivité du travail, des surfaces et des animaux, s'affrontent aux nouvelles demandes sociales de préservation de l'environnement et des espaces ruraux. Dans les pays en développement, et notamment dans les plus pauvres d'entre eux, au contraire, la production fait défaut, les déficits alimentaires s'accroissent, tandis qu'une part importante de la population, notamment en milieu rural, souffre de faim ou de malnutrition. La crise environnementale et sociale des agricultures du Nord, la crise alimentaire et sociale des agricultures du Sud se traduisent désormais dans une exigence nouvelle : celle d'un développement durable de l'activité agricole.

Avant même d'avoir entrepris de définir ce nouveau concept de « développement durable ", on entrevoit sa complexité, puisque l'exigence de durabilité pourrait impliquer au Nord une baisse de l'intensification des systèmes de production, tandis qu'elle impliquerait au Sud une augmentation de la productivité des surfaces, du travail et de la production, pour faire face aux besoins alimentaires les plus urgents des populations concernées.

\footnotetext{
* Ce texte est issu de la conférence prononcée dans le cadre de l'Académie d'Agriculture, le 15 juin 2005, à Paris.
} 
Ces contradictions soulignent la difficulté $d^{\prime}$ 'une réflexion sur la manière dont les politiques actuelles permettent ou non d'atteindre cet objectif de développement durable. La première difficulté surgit dans la définition même du concept de « développement durable » car il n'existe pas aujourd'hui de définition précise universellement partagée. La définition la plus usuelle est celle qu'en a donnée la commission Brundtland en 1987, reprise en 1992 à la Conférence de Rio :

- « Un développement qui répond aux besoins du présent sans compromettre la capacité des générations futures de répondre à leurs propres besoins. »

Pour utile que soit cette définition, qui introduit explicitement la nécessité d'un raisonnement intergénérationnel à long terme en rupture avec les raisonnements d'optimisation économique à court terme, elle reste encore trop générale pour être opératoire, ce qui limite fortement le caractère contraignant d'un développement « plus durable ». Ainsi que le soulignent les économistes de l'environnement, "c'est son ambiguiité qui fait le succès du développement durable. Pour les écologistes, le volume de production doit être supportable pour l'écosystème. L'interprétation dominante au contraire est de faire durer le développement, c'est-à-dire la croissance : pourvu que ça dure » $[1,2]$.

De fait, il n'est pas de réalisation, d'innovation technique, autoroute, centrale nucléaire, viaduc, OGM... qui ne soit considérée aujourd'hui comme une contribution décisive à un développement plus durable. Et, en la matière, les politiques agricoles contemporaines ne sont pas en reste.

Concept flou, le développement durable n'en est que plus utilisé. Avec d'autres concepts comme la « multifonctionnalité » ou « l'économie sociale de marché », il a le mérite de permettre des consensus politiques larges, sans poser les délicates et conflictuelles questions du partage des responsabilités et des moyens. Le développement durable reste donc aujourd'hui une intention. Passer au stade opératoire (stade nécessairement plus contraignant) nécessite en effet l'élaboration d'indicateurs précis, permettant d'apprécier le degré de durabilité des différentes possibilités de choix. II ne peut s'agir que d'indicateurs complexes, construits à partir de critères multiples, qui couvrent les trois axes usuellement admis de la durabilité : durabilité économique, durabilité sociale, durabilité environnementale.

Dans le domaine de l'évaluation globale de l'activité d'un pays ou d'un secteur d'activité, il existe de nombreux critères d'évaluation macroéconomique, tels que le PIB par habitant, le taux de croissance du PIB, le taux $d^{\prime}$ 'inflation ou le taux de rentabilité des investis- sements, l'indice d'évolution des valeurs boursières, etc. qui peuvent constituer autant d'outils d'appréciation de la « durabilité économique ». Dans le domaine de l'évaluation de la durabilité sociale du développement, il existe aussi des indicateurs multicritères tels que I'indice de développement humain (IDH) mis au point par le Programme des Nations Unies pour le Développement. Bien que d'un grand intérêt, ce type d'indicateur reste encore aujourd'hui peu utilisé, dans l'évaluation des politiques de développement. En matière d'environnement, la situation est encore plus indigente. Un certain nombre de travaux expérimentaux existent depuis le début des années 70, mais il n'existe pas de système harmonisé de comptabilité environnementale (M. Weber, 1990). Depuis 1974, la comptabilité nationale a connu de nombreuses adaptations, notamment avec la mise en place en 1995 d'un système européen des comptabilités (SEC 95) qui reprend très largement le système proposé par I'ONU, dont les pays membres doivent $s^{\prime}$ inspirer. Mais, parmi les améliorations envisagées, toutes n'ont pas été retenues. "L'idée d'apporter des corrections au PIB, pour prendre en compte les relations entre l'activité économique et la nature ne s'est pas concrétisée. La réflexion sur la valorisation de ces relations est apparue encore insuffisamment mûre.» (D. Temam, 1998). En dépit des nouvelles aspirations à un développement plus durable, il faut donc bien prendre acte que c'est encore l'augmentation du PIB et de la production marchande qui reste aujourd'hui l'indicateur privilégié du développement (réduisant du même coup le concept pluridimensionnel du développement au critère unidimensionnel de la croissance).

Dans le domaine qui nous intéresse plus particulièrement, l'agriculture, la réflexion n'est guère plus avancée, en dépit du rôle moteur que les politiques entendent désormais faire jouer à l'agriculture, pour la promotion d'un développement durable. II existe bien en France un dispositif solide d'évaluation annuelle des performances de la branche, piloté par la Commission nationale des Comptes de I'Agriculture. Ce dispositif permet de disposer chaque année d'informations diversifiées sur les produits, les charges et les résultats de l'activité agricole, par région et par orientation de production. II donne également des informations riches sur les transferts et les dépenses publiques pour l'agriculture, les comptes sociaux de la branche, les comparaisons avec les autres branches. En revanche, aucun indicateur, même partiel, n'est produit pour évaluer la durabilité de l'activité agricole. Par rapport à l'année précédente, l'activité agricole a-t-elle consommé plus ou moins d'énergie fossile, plus ou moins de pesticides?
A-t-elle rejeté plus ou moins de nitrates ou de gaz à effet de serre dans l'environnement? Autant d'indicateurs qui pourraient donner un aperçu de la durabilité du développement agricole et qui font encore aujourd'hui défaut. Même partiels, ils permettraient une évaluation plus nuancée du développement de l'agriculture, que ne le permettent la seule valeur ajoutée par actif ou le seul résultat net par actif familial, qui polarisent aujourd'hui toute l'attention des professionnels agricoles.

Reste que le développement durable de l'agriculture est devenu, en Europe, une préoccupation explicite de la politique agricole ainsi que I'a arrêté le Conseil européen de Göteborg: "Sustainable development [...] require dealing with economic, social and environmental policies in a mutually reinforcing way [...]. The CAP should, among its objectives, contribute to achieve sustainable development" (Conseil européen de Göteborg, juin 2001). L'urgence de cette préoccupation n'est toutefois pas telle qu'elle ait conduit les décideurs politiques à commander aux scientifiques la mise au point de la batterie diversifiée de critères nécessaire à une évaluation des effets de la politique agricole en matière de développement durable. Autant dire que la discussion de l'impact des politiques actuelles sur le développement durable en agriculture ne peut être aujourd'hui abordée que de manière très fragmentaire, à partir $d^{\prime}$ analyses qualitatives ponctuelles, faute d'outils d'évaluation adéquats.

\section{De la durabilité économique de l'agriculture}

Les critères d'évaluation économiques sont, nous l'avons rappelé, les plus nombreux et les plus facilement accessibles. À défaut d'analyser la durabilité du développement agricole dans l'ensemble de ses composantes, nous nous proposons ici d'en analyser la durabilité dans sa composante la plus facile à évaluer : la composante économique.

Pour illustrer notre propos, nous avons réuni dans les tableaux qui suivent un certain nombre de résultats élaborés à partir d'un traitement spécifique des données du Réseau d'Information Comptable disponibles pour l'année 2003 [3]. La population couverte par l'échantillon RICA concerne les exploitations identifiées comme professionnelles (c'est-à-dire employant au moins 0,75 unités de travail par an), soit 379000 exploitations sur un total de 600000 exploitations environ. Quoique ne constituant qu'une partie de la population totale, ces exploitations professionnelles représentent l'essentiel de la production française, puisqu'elles occupent plus de $90 \%$ de la SAU du pays ( 25,8 millions d'hectares, sur un total de 28,3 millions d'hectares). 
Tableau 1. Ensemble des exploitations (\% d'exploitations selon RCAI/UTAF).

\begin{tabular}{|lcccc|}
\hline Classe de taille $(\mathbf{1})$ & $<\mathbf{0} €$ & $\mathbf{0}$ à $\mathbf{1 3} \mathbf{8 0 0} €$ & $>\mathbf{1 3 8 0 0} €$ & Total \\
\hline$<40$ UDE & 13 & 46 & 40 & 100 \\
40 à 80 UDE & 8 & 33 & 58 & 100 \\
$>$ 80 UDE & 9 & 20 & 71 & 100 \\
Toutes exploitations & 10 & 34 & 55 & 100 \\
\hline
\end{tabular}

(1) mesurée en marge brute standard (MBS) traduite en unités de dimension économique = UDE. 1 UDE $=1200 €$ de marge brute standard, équivalent à 1,5 ha de blé environ (80 UDE correspond environ à 120 ha équivalent blé).

Les exploitations professionnelles (tableau 1) ont été classées en trois catégories, selon leurs performances économiques, ici évaluées à partir du résultat courant avant impôt par unité de travail familial (RCAI/UTAF). Les bornes des classes correspondent respectivement à un RCAI/UTAF nul, et à un RCAI/UTAF de $13800 €$, qui équivaut au montant du SMIC annuel brut. Bien que le résultat courant avant impôt par actif ne constitue pas un indicateur de revenu directement comparable au revenu d'un salarié, nous avons gardé cette référence au SMIC comme ordre de grandeur pour mieux illustrer notre propos.

Premier constat: un nombre important d'exploitations professionnelles, toutes orientations de production confondues, dégagent un résultat par actif faible, négatif ou inférieur au SMIC. Cette proportion est particulièrement forte, de l'ordre de $60 \%$, dans les petites exploitations inférieures à 40 UDE. Elle diminue certes avec la dimension économique, mais elle reste encore importante dans les grandes exploitations de plus de 80 UDE (29\%). Cela signifie donc que le lien entre la dimension économique et le résultat existe bien, mais que la dimension économique n'est qu'un facteur parmi beaucoup d'autres susceptibles d'expliquer les performances économiques.

Par ailleurs, le nombre d'exploitations dégageant un résultat par actif (RCAI/UTAF) négatif peut surprendre. Ce résultat doit s'interpréter à partir des modalités de calcul du résultat courant avant impôts, qui déduit des produits I'ensemble des charges non exceptionnelles, y compris I'amortissement des matériels et bâtiments. C'est donc un indicateur qui évalue le solde disponible sous condition de renouvellement de l'appareil de production. Lorsque l'exploitation ne renouvelle pas ses équipements et ses bâtiments, les dépenses effectives sont inférieures aux amortissements calculés (voire nulles) et l'excédent de trésorerie disponible pour les prélèvements familiaux supérieur au RCAI. À court terme, on peut donc vivre avec un RCAl négatif, en hypothéquant la capacité de renouvellement à moyen ou long terme de l'exploitation. Au-delà des aléas conjoncturels, qui peuvent grever les résultats d'une année donnée, les exploitations qui dégagent des RCAI négatifs ou très faibles sont des exploitations en voie de disparition, qui se maintiennent temporairement, en consommant leur capital. Leur disparition effective se concrétise le plus souvent au moment du changement de génération, par l'absence de succession lors du départ à la retraite des exploitants en place. C'est le mécanisme bien rôdé de l'exode agricole, qui fonctionne avec une grande régularité depuis un demi-siècle, et que les réformes successives de la PAC en 1984, en 1992, en 1999 ou en 2003 n'ont pas altéré. Ce constat soulève une première interrogation concernant la durabilité de l'activité agricole. Au sens strict, l'activité agricole ne serait pas durable, en France, puisque le potentiel humain en activité dans l'agriculture diminue. Or, les différentes politiques qui se sont succédé se sont parfaitement accommodées de l'exode agricole, quand elles ne l'ont pas accompagné. Cela conduit à compléter notre concept de durabilité, en y incluant une dimension politique, qui renvoie à l'acception du terme, dans le sens littéral de sa traduction : un développement «soutenable ». Jusqu'à quel niveau l'exode agricole reste-t-il soutenable? Difficile de répondre scientifiquement à une telle question, qui relève à l'évidence de choix politiques. La notion de durabilité apparaît donc relative. Est durable ce qui est aussi politiquement soutenable. Ce constat pourrait conduire à une quatrième dimension de la durabilité, à côté de sa dimension économique, sociale et environnementale: celle de son acceptabilité politique.

\section{Une agriculture sous perfusion}

À partir des tableaux qui précèdent, nous avons élaboré plusieurs tableaux dérivés, afin d'analy-
Tableau 2. Ensemble des exploitations professionnelles (en \%). ser le poids des aides publiques dans la formation des revenus des agriculteurs, en calculant cette fois un résultat courant avant impôt par actif familial, hors aides publiques directes (RCAI - aides directes/UTAF).

Concernant la répartition des aides publiques, la réforme de juin 2003 n'entraîne pas de modifications sensibles par rapport à la situation observée dans le RICA 2003, sauf en élevage laitier. Pour dresser les tableaux qui suivent, nous avons donc simulé, en production laitière, l'effet anticipé de la réforme de l'OCM lait prévue dans les faits pour 2005, sur les résultats 2003. L'analyse qui suit concerne donc une estimation de l'impact des soutiens après mise en œuvre de la réforme de juin 2003 (tableau 2).

On vérifie sans surprise que les aides jouent un rôle essentiel dans la formation des résultats économiques des exploitations professionnelles. En effet, sans aides, plus de la moitié des exploitations dégagent un revenu négatif, et plus de $80 \%$ un résultat courant par actif inférieur au SMIC. Et ce constat concerne I'ensemble des exploitations, toutes orientations de production confondues. II inclut notamment les productions comme la viticulture ou I'horticulture qui dépendent peu des aides directes. De fait, dans les orientations de grande culture et d'élevage qui dépendent le plus des aides, le nombre d'exploitations capables de dégager un RCAI/UTAF sans aides positif et supérieur au SMIC est encore plus faible (tableau 3).

L'analyse détaillée par classe de taille au sein de chaque orientation de production montre, en outre, que l'effet «dimension économique » ne modifie que faiblement la distribution des résultats. En effet, les grandes exploitations étant généralement celles qui bénéficient le plus des aides directes, la suppression des aides pénalise plus lourdement leur revenu que celui des exploitations de tailles plus modestes. À l'extrême, en élevage bovin viande, la dimension accentue l'effet dépressif de la suppression des aides (tableau 4).

Ces résultats laissent facilement entrevoir l'effet de décomposition des structures et de démobilisation de productions auquel conduirait la suppression des aides directes à l'agriculture que les économistes libéraux les plus convaincus appellent de leurs vœux. En effet, dès lors que les grandes unités de production ne se révèlent pas

\begin{tabular}{|lcccc|}
\hline Dimension & \multicolumn{3}{c|}{ (RCAI - aides directes) / UTAF } & Total \\
\cline { 2 - 4 } économique & $<\mathbf{0} €$ & $\mathbf{0}$ à $\mathbf{1 3} \mathbf{8 0 0} €$ & $>\mathbf{1 3} \mathbf{8 0 0} €$ & \\
\hline$<40$ UDE & 60 & 30 & 10 & 100 \\
40 à 80 UDE & 60 & 25 & 15 & 100 \\
$>80$ UDE & 53 & 20 & 27 & 100 \\
Total & 58 & 26 & 16 & 100 \\
\hline
\end{tabular}


Tableau 3. Ensemble des exploitations professionnelles (en \%).

\begin{tabular}{|lcccc|}
\hline Orientation de & \multicolumn{3}{c|}{ (RCAI - aides directes) / UTAF } & Total \\
\cline { 2 - 4 } production & $<\mathbf{0} €$ & $\mathbf{0}$ à $\mathbf{1 3} \mathbf{8 0 0} €$ & $>\mathbf{1 3} \mathbf{8 0 0} €$ & \\
\hline Grandes cultures & 64 & 21 & 11 & 100 \\
Bovin lait & 60 & 36 & 4 & 100 \\
Bovin viande & 77 & 20 & 3 & 100 \\
Ovins caprins & 76 & 21 & 3 & 100 \\
Polyculture-élevage & 64 & 26 & 10 & 100 \\
Granivores & 38 & 40 & 21 & 100 \\
Autres & 19 & 28 & 53 & 100 \\
Ensemble & 58 & 26 & 16 & 100 \\
\hline
\end{tabular}

Tableau 4. Répartition des exploitations professionnelles Bovins viande (en \%).

\begin{tabular}{|lcccc|}
\hline Dimension & \multicolumn{3}{c|}{ (RCAI - aides directes) / UTAF } & Total \\
\cline { 2 - 4 } économique & $<\mathbf{0} €$ & $\mathbf{0}$ à $\mathbf{1 3 ~ 8 0 0 €}$ & $>\mathbf{1 3} \mathbf{8 0 0} €$ & \\
\hline$<40$ UDE & 75 & 22 & 4 & 100 \\
40 à 80 UDE & 82 & 15 & 3 & 100 \\
$>80$ UDE & 83 & 17 & 1 & 100 \\
\hline
\end{tabular}

sensiblement plus performantes, sans aides directes, que les plus petites, il est illusoire d'espérer qu'une restructuration accélérée des exploitations puisse permettre de faire face à la suppression des aides. Même en supposant que toutes les orientations de production soient organisées en exploitations de grande dimension (plus de 80 UDE), I'activité agricole ne permettrait de dégager un résultat positif (RCAI) que sur $35 \%$ des exploitations en grandes cultures (soit $30 \%$ des surfaces), que sur $35 \%$ des exploitations en bovins lait (soit $32 \%$ des surfaces), que sur $17 \%$ des exploitations en bovins viande (soit $12 \%$ des surfaces)...

Au total, même dans l'hypothèse d'une concentration de la production dans des exploitations de grande dimension économique, c'est, en cas de suppression totale des aides directes, $67 \%$ de la SAU française qui se trouverait détenue par des exploitations dégageant un résultat négatif et $85 \%$ des surfaces qui se trouveraient détenues par des exploitations dégageant un résultat par actif (RCAI/UTAF) inférieur à $13800 €$.

Sans entrer dans des scénarios bien compliqués, ces chiffres laissent entrevoir qu'une suppression brutale des aides conduirait à une démobilisation impressionnante de la production par élimination des exploitations les moins rentables. Et cette démobilisation des surfaces resterait très importante, même en cas de restructuration poussée des exploitations et de concentration de la production dans de grandes exploitations (> 80 UDE). Corrélativement, le double mécanisme de restructuration et de démobilisation de la production conduirait à une population résiduelle d'exploitations de
I'ordre de 115000 (toutes orientations de production confondues) pour une population initiale d'exploitations professionnelles de $379000(-70 \%)^{3}$. Dans les orientations de production les plus dépendantes des aides, la saignée démographique serait plus importante encore. En grandes cultures, les effectifs passe$(-76 \%)$, en production bovins-viande les effectifs passeraient de 42000 à 2800 exploitations $(-93 \%)$, et en production ovine et caprine les effectifs passeraient de 21200 à 3700 (- $82 \%)$. Même si ces estimations restent grossières, une conclusion s'impose : sans aide publique, I'agriculture française $n^{\prime}$ est absolument pas durable d'un simple point de vue économique. II est donc tout à fait erroné de prétendre justifier les aides pour des motifs environnementaux ou sociétaux. Indépendamment de toute production de services environnementaux ou sociétaux, les aides sont indispensables à la pérennité de l'activité économique marchande des exploitations.

À partir de là, faut-il conclure que l'agriculture française, dans son immense majorité, n'est pas économiquement rentable et que c'est une

\footnotetext{
${ }^{3}$ Ces résultats sont obtenus en faisant l'hypothèse que la surface actuelle de chaque OTEX est occupée uniquement par des exploitations de plus de 80 UDE ( $d$ 'ou une première réduction des effectifs de - $41 \%$ sur le total des exploitations professionnelles), et qu'après cette restructuration, toutes les exploitations restantes dégageant un RCAI négatif disparaissent (deuxième réduction des effectifs de $48 \%$ ) soit une réduction globale de $72 \%$ ).
} raient de 97000 à 23000 exploitations agriculture qui ne peut subsister que grâce à la perfusion continue, année après année, d'aides publiques considérables ? Faut-il enfin admettre que la PAC a soutenu des choix antiéconomiques, conduisant à soutenir à grands frais, pendant quatre décennies, une agriculture structurellement inefficace, incapable malgré des efforts importants d'aide à la modernisation d'assurer seule sa durabilité économique? Puisqu'encore aujourd'hui, deux tiers des surfaces françaises produisent à perte sans aide publique, n'est-ce pas le signe incontestable qu'il convient d'abandonner définitivement la chimère de l'autosuffisance communautaire (encore plus celle de la compétitivité internationale) pour s'approvisionner, dans des conditions infiniment plus avantageuses pour les finances publiques, à moindre coût sur les marchés internationaux ? C'est bien le constat auquel semble aboutir un certain nombre de hauts fonctionnaires européens... Avant de se résigner à une telle conclusion, nous proposons d'ouvrir le débat sur une nouvelle problématique : est-ce l'agriculture qui n'est pas durable ou est-ce la politique agricole commune qui est devenue économiquement insoutenable?

\section{Théorie économique et marchés agricoles : les illusions de la concurrence}

Suite aux réformes successives entreprises depuis 1992, la politique agricole que nous connaissons aujourd'hui conduit à alimenter une part essentielle du revenu des agriculteurs (souvent plus de $100 \%$ ) par des aides directes. La justification de cette politique résulte de I'application de principes économiques simples : en concurrence parfaite, il est nécessaire, pour optimiser le bien-être collectif, et tout particulièrement celui des consommateurs, de décloisonner les marchés, de désarmer les protections aux frontières. II est donc légitime, souhaitable, nécessaire, d'aligner les prix domestiques sur les prix mondiaux. II faut pour cela rompre définitivement avec le soutien des prix, quitte à donner aux producteurs des aides compensatoires «découplées » c'est-à-dire indépendantes de toute activité de production. Nous ne reprendrons pas ici la liste des hypothèses du modèle de concurrence parfaite qui permet de conduire à ce résultat [4]. Rappelons simplement qu'il s'agit d'un modèle d'équilibre général, qui concerne l'ensemble des marchés. II suffit donc qu'un seul marché ne soit pas parfaitement concurrentiel pour que, par contamination des interférences d'un marché à l'autre, l'équilibre général (équilibre entre I'offre et la demande de chaque bien), s'il existe, ne conduise plus à l'optimum (équilibre général contraint). Rappelons aussi que les auteurs du modèle ont eux-mêmes montré 
qu'en cas de déséquilibre initial, les tâtonnements successifs des agents pour approcher les prix d'équilibre ne conduisaient pas nécessairement à l'équilibre recherché [5].

Mais le problème qui nous est posé n'est pas de discuter la validité du modèle, construit selon une axiomatique parfaitement claire et rigoureuse. La question qui nous est posée est de confronter l'adéquation des hypothèses à la réalité du fonctionnement de marchés agricoles, et partant, l'adéquation de la PAC aux exigences d'un développement agricole durable. On admet assez facilement, en physique, que la loi de gravitation découverte par Newton (chute des corps selon un mouvement uniformément accéléré) est vérifiée sous certaines hypothèses (dans un espace sous vide notamment). On admet facilement que, dans un espace rempli par un fluide, par exemple I'air (changement des conditions d'expérience), les phénomènes prennent une autre allure. La prise en compte des frottements et des mouvements du fluide permet alors $d^{\prime}$ expliquer pourquoi les avions volent et pourquoi les parachutistes ne s'écrasent pas tous au sol. Et ce n'est pas une insulte à Newton que de faire ce type de constat. En économie agricole, il en va un peu de la même manière. Le fonctionnement des marchés est sujet à de nombreuses contraintes, de nombreux frottements. Les imperfections des marchés sont d'origines multiples: information imparfaite, délais d'ajustement, incertitude et risques, aléas biologiques et climatiques, etc. La rationalité économique des agents $s^{\prime}$ en trouve substantiellement changée. Tout comme le parachutiste apprend à planer, les agents économiques apprennent à se mouvoir dans un espace économique dense, où les frottements sont multiples. Ils apprennent à jouer avec le temps, à spéculer, à jouer avec la monnaie, à faire des paris sur l'avenir. Ils construisent aussi des stratégies pour se protéger des risques, pour partager l'incertitude. Ils apprennent à construire des formes d'organisation et d'actions collectives pour pallier l'inefficacité de leurs actions isolées, etc.

La nature même des marchés s'en trouve profondément modifiée, si bien que la théorie initiale perd toute signification normative, pour orienter les choix de politique agricole. En effet, sans revenir longuement sur les fondements même de l'économie rurale, on rappellera qu'en agriculture les conditions de fonctionnement des marchés sont particulièrement contraignantes (contraintes climatiques et naturelles, marchés des facteurs de production incomplets, faible élasticité de la demande et instabilité des prix, etc.), si bien qu'il devient illusoire d'espérer que les principes d'une concurrence libre et non faussée puissent effectivement constituer une réponse appropriée aux exigences de durabilité de l'activité agricole. Mais le constat semble encore difficile à admettre, à voir l'obstination des politiques agricoles à vouloir appliquer les principes d'une concurrence parfaite au pilotage de marchés qui fonctionnent pourtant selon une tout autre logique.

\section{Le marché mondial : réalité empirique et fiction théorique}

Ainsi le prix mondial constitue-t-il encore le pivot des politiques agricoles contemporaines, le prix de référence qui sert à étalonner et à évaluer toute mesure de soutien des marchés. Tout prix domestique supérieur au prix mondial est ainsi considéré comme un prix abusivement soutenu. Les mots sont ici piégés. Certes, il existe bien un «marché mondial » au sens usuel qu'emploient les opérateurs, mais du point de vue de la théorie économique, aucune des hypothèses nécessaires à l'existence d'un marché mondial concurrentiel n'existe. L'espace international n'est pas un espace économique homogène. Les marchés restent cloisonnés. Les échanges internationaux ne concernent, faut-il le rappeler, qu'une part réduite des volumes produits et échangés. II n'y a donc pas un marché mondial des produits agricoles, mais des marchés nationaux ou régionaux qui se connectent entre eux pour échanger des volumes résiduels (10 à $15 \%$ de la production). Certes, il y a bien une offre et une demande à l'international, mais elle ne concerne qu'une fraction limitée de l'offre et de la demande mondiales. Le cours des transactions internationales ne peut donc nullement prétendre s'imposer comme prix de référence pour les transactions qui s'effectuent hors du « marché mondial » et qui constituent l'essentiel des transactions. Supposons un instant que I'INSEE, pour calculer l'indice du coût de la vie, se fonde sur les prix relevés fin janvier, dans les grandes surfaces, à la fin de la période des soldes. Il y a bien un « marché » avec une offre et une demande, qui fonctionne à cette période. Qui oserait toutefois prétendre que I'indice ainsi calculé refléterait sérieusement l'indice du coût de la vie?

Or, toutes les constructions de politique agricole contemporaine reposent sur le postulat selon lequel le cours des transactions internationales représente le prix d'équilibre qui devrait s'établir sur un marché de concurrence parfaite, ce qui est une pure chimère sans aucun fondement théorique. De fait, le cours des transactions internationales ne couvre pas les coûts de production des agriculteurs de la plupart des régions du monde et seules de substantielles aides directes, en Europe comme aux États-Unis, leur permettent de maintenir une activité de production. En matière de production laitière, la situation paraît encore plus caricaturale. L'OCDE estime par exemple que la référence, pour déterminer le « prix mondial » des produits laitiers, est le prix à l'exportation de la Nouvelle-Zélande qui représente $2,6 \%$ de la production mondiale et dont les exportations représentent environ $2 \%$ de cette même production. Qui peut raisonnablement accepter que ce prix-là, l'un des plus bas du monde serve de référence pour orienter l'ensemble de la production du globe ? À ce prix, c'est la quasi-totalité de producteurs des autres pays qui est condamnée à disparaître (sauf bien entendu soutiens directs massifs des pouvoirs publics). Et qui peut raisonnablement croire que la Nouvelle-Zélande est en mesure $d$ 'assurer seule l'approvisionnement en lait de I'ensemble du reste du monde?

Qui peut raisonnablement croire qu'on peut se passer de la production céréalière américaine et européenne pour assurer la sécurité alimentaire de la planète?

\section{Des politiques construites sur des hypothèses fragiles}

Pourtant, c'est bien à partir de ces prix-là qu'on évalue désormais, à l'OCDE et à I'OMC, les niveaux de soutien et les fameux Équivalent Soutien à la Production (ESP). Par un renversement complet de la logique, on considère ainsi que l'écart entre les prix domestiques à la production (jugés abusivement soutenus) et le prix mondial (en l'occurrence le prix à l'exportation néo-zélandais pour les produits laitiers) représente un soutien indirect aux producteurs, qui vient $s^{\prime}$ ajouter aux soutiens directs versés par les pouvoir publics pour calculer les fameux ESP. On n'hésite pas pour cela à additionner des dépenses bien réelles, avec des soutiens tout à fait virtuels. D'ailleurs, la logique de l'argumentation change selon le contexte. S'il $s$ 'avère que des prix soutenus aux producteurs augmentent les ESP et pénalisent les consommateurs, à l'inverse une baisse des prix devrait diminuer les soutiens à la production et augmenter le bien-être des consommateurs. Dans ce cas, des pays comme la Grande-Bretagne et I'Allemagne ne devraient avoir aucune raison de se plaindre du coût budgétaire de la PAC, dans la mesure où les dernières réformes et les baisses de prix substantielles qui les ont accompagnées ont contribué à augmenter significativement le bien-être de leurs consommateurs. Malheureusement pour la France, nos partenaires européens sont plus terre-à-terre et savent faire la différence entre une dépense budgétaire réelle et une économie virtuelle. Il est par ailleurs paradoxal de voir les instances internationales comme I'OCDE et I'OMC s'intéresser avec tant d'attention à l'évaluation 
des ESP et des aides « distorsives » (celles qui faussent les jeux de la libre concurrence) et rester totalement muettes sur un facteur essentiel de distorsion des échanges : les fluctuations des parités monétaires et le traitement inégal de l'endettement. Cela souligne au passage I'inégal pouvoir des instances internationales: le FMl, qui traite des questions monétaires et d'endettement, s'autorise sans restriction des interventions dans le domaine commercial, en soumettant notamment ses plans d'ajustement structurel à l'exigence d'une insertion accrue dans l'échange international. En revanche I'OMC ne traite que de questions commerciales et ignore totalement l'effet de distorsion des taux de change sur la concurrence libre et non faussée.

Quant à l'inégalité de traitement de l'endettement, deux chiffres permettent d'en mesurer l'ampleur. L'endettement total de l'ensemble des pays en développement s'élève à 2450 milliards de dollars (1 600 milliards de dettes publiques et 850 milliards de dettes privées), soit douze fois moins que l'endettement global des États-Unis qui s'élève à 29000 milliards de dollars (7 400 milliards de dettes publiques et 21600 milliards de dettes privées) [6]. Or, il est clair que le FMI n'applique pas les mêmes traitements aux uns et aux autres. L'effet indirect sur les échanges est pourtant essentiel, dans la mesure où les pays en développement, fortement endettés et astreints au remboursement de leur dette, sont obligés d'exporter des matières premières agricoles à $n^{\prime}$ importe quel prix pour se procurer les recettes en devises nécessaires pour honorer leurs échéances. L'endettement du Brésil, par exemple, permet aisément d'expliquer son agressivité à l'exportation. Les États-Unis, quant à eux, s'endettent massivement pour consommer. Ils bénéficient du statut enviable de « consommateurs en dernier ressort » de l'économie mondiale, puisque leur surconsommation apparaît comme un stimulant essentiel de la demande internationale. $N^{\prime} y$ a-t-il pourtant pas quelques bonnes raisons de penser qu'un développement économique plus durable passe d'abord par la satisfaction des besoins des pays les plus pauvres, plutôt que par l'augmentation du gaspillage des plus riches?

Qu'il s'agisse de la référence au prix mondial, des modalités de calcul des ESP ou de l'ignorance des questions monétaires, les ingrédients à partir desquels se recomposent les politiques agricoles contemporaines ne paraissent pas parfaitement pertinents pour assurer un développement économique durable de l'agriculture. II semble qu'il en aille de même avec le découplage, dernier avatar des réformes contemporaines des politiques agricoles.

\section{Le découplage : quelques questions à partir de l'exemple américain}

Le découplage est un sous-produit de la logique du raisonnement économique évoqué précédemment. Puisque le prix mondial est le prix de référence, comment résoudre la contradiction entre la baisse des prix et le maintien de l'activité productive ? On l'a vu avec l'exemple français, mais d'autres travaux menés aux États-Unis conduisent aux mêmes conclusions $[7,8]$, la production, même dans les zones les plus favorisées des deux pays, ne pourrait pas se maintenir sans de substantielles aides publiques, découplées ou non. La notion de découplage reste d'ailleurs très ambiguë. D'une part, elle laisse entendre que les aides découplées, à la différence des politiques de prix, sont ciblées sur des objectifs spécifiques (multifonctionnalité, durabilité) alors qu'elles restent en fait essentiellement liées à l'activité productive marchande. D'autre part, la réalité du classement des aides dans les différentes boîtes résulte d'abord d'un arbitrage politique bien plus que d'une réalité économique, pour la raison simple qu'il est facile, en théorie, de définir le découplage, mais qu'il est beaucoup plus difficile, dans la pratique, de discerner l'impact réel d'une aide sur l'orientation de la production et des marchés. D'une manière générale, les économistes reconnaissent aujourd'hui qu'il n'est pas d'aide, si découplée qu'elle se prétende, qui n'ait un effet distorsif indirect sur les échanges, au point d'ailleurs qu'un certain nombre des partenaires de I'UE et des USA à l'OMC, remettent en question la classification des aides actuellement en vigueur.

Sur le sujet, l'exemple américain est riche d'enseignement. D'abord parce que les ÉtatsUnis ont découplé une partie de leurs aides en 1996, et que, suite à l'expérience des années 99, 2000 et 2001 (multiplication des aides d'urgence pour enrayer la baisse des revenus agricoles), ils ont réintroduit des aides couplées dans leur loi de programmation agricole de 2002, sous une forme nouvelle désormais appelée paiements contracycliques d'une part, en renforçant d'autre part les aides couplées liées aux dispositifs des marketing loan. La figure 1 illustre les modalités actuelles de mise en œuvre des soutiens au maïs aux États-Unis, selon les dispositions de la loi de programmation agricole de 2002 [9].

L'aide globale que touche le producteur se décompose comme suit :

- La première partie du soutien couvre l'écart entre le prix de base garanti (effective target price) et le prix final garanti au producteur (target price). Ces deux prix sont définis de manière strictement administrative et de manière a priori indépendante du marché. Le paiement du différentiel de prix (découplé du marché) s'applique à une production historique indépendante de la production réelle (donc également découplée), ce qui permet de considérer que ce paiement direct est totalement découplé (c'est en fait la reprise, dans la Loi-programme agricole de 2002, des paiements découplés institués dans la loiprogramme précédente de 1996).

- La seconde partie du soutien couvre l'écart entre le prix moyen de marché (market price) et le prix de base garanti à la production (effective target price) lorsque le prix moyen de marché est supérieur au prix minimum garanti de commercialisation (loan rate). C'est le paiement contracyclique, qui dépend du niveau de prix, mais qui s'applique à une production historique (rendements historiques $x$ surfaces historiques) et non à la production réelle, et que l'on considère pour cela comme «semidécouplé ». Lorsque le prix moyen de marché tombe au-dessous du prix minimum de commercialisation garanti le paiement contracyclique couvre la totalité de la différence entre le prix de base garanti (effective target price) et le prix minimum de commercialisation garanti (loan rate).

- Une troisième forme de soutien intervient, lorsque le prix réel de marché tombe en dessous du prix de commercialisation garanti (loan rate). Elle couvre alors l'écart entre le prix réel de marché, observé chaque semaine dans chaque comté (posted county price) et le prix de commercialisation garanti (loan rate). La compensation s'applique aux volumes réels commercialisés (mécanisme du marketing loan et des loan defficiency payment), et est, de ce fait, considérée comme totalement couplée.

À partir de ce mécanisme de soutien, on peut mettre en évidence le caractère tout à fait artificiel de la notion de découplage. II suffit en effet de baisser le prix de base garanti (effective target price) pour le rapprocher du prix de commercialisation garanti (loan rate), pour transformer le paiement contracyclique semidécouplé en paiement direct totalement découplé... (figure 2). Autant dire que le découplage procède plus, en réalité, de l'habilité de la négociation politique que de la rigueur de I'analyse économique, ce qui devrait conduire les économistes à une grande modestie.

Cette baisse du prix de base garanti, et l'augmentation du paiement direct découplé qui l'accompagne, ont toutefois un inconvénient : ils assurent, en cas de hausse des prix, une rente aux producteurs qui cumulent alors le bénéfice de prix rémunérateurs et le bénéfice des aides découplées, acquises quoi qu'il arrive. (C'est d'ailleurs ce qui s'est passé en 1996 et 1997, ce qui explique que le découplage a été au départ parfaitement bien accepté par les 


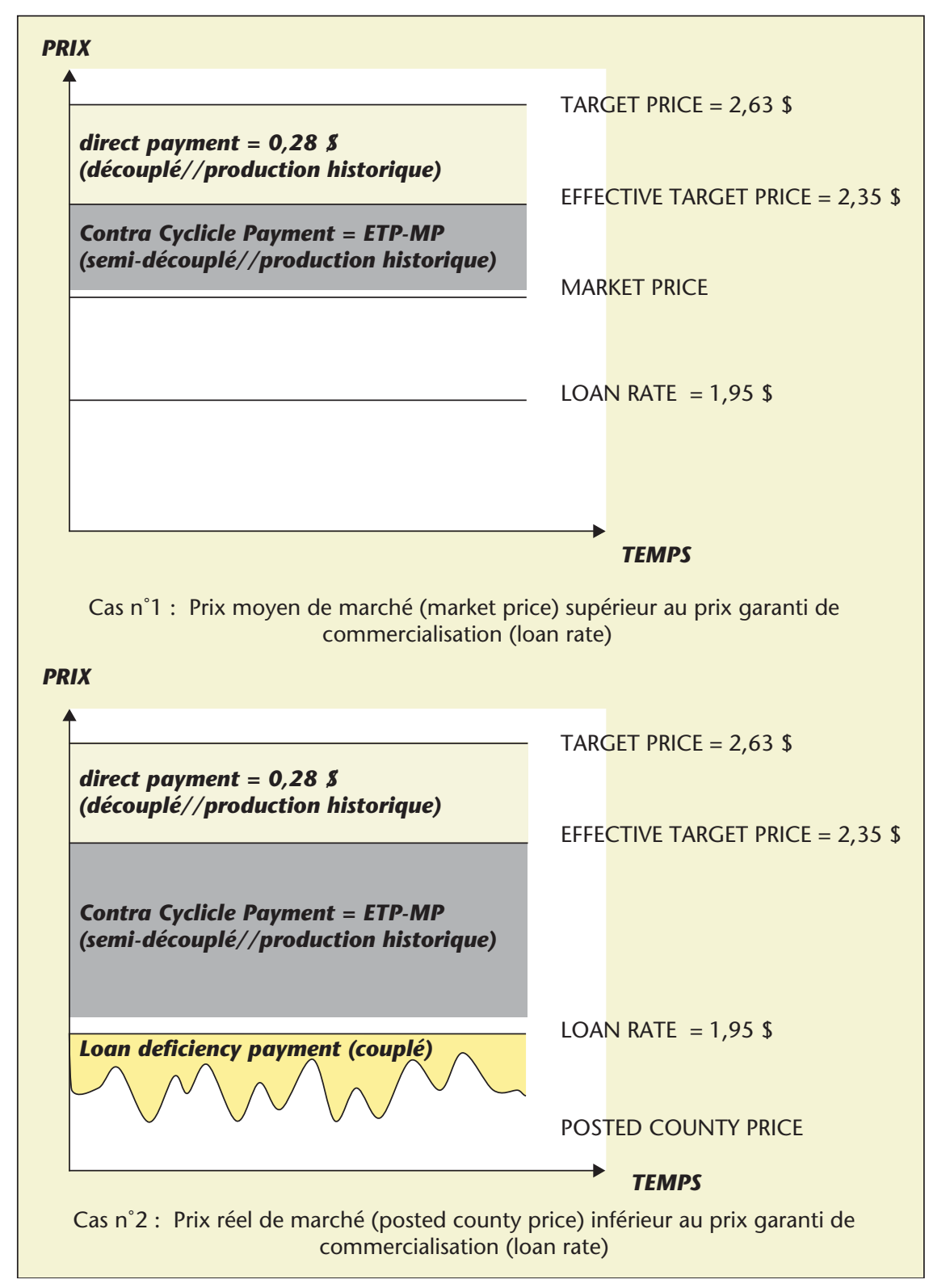

Figure 1. Le dispositif de soutien des prix aux États-Unis (FSRIA de 2002, exemple du maiis).

producteurs agricoles américains, tout comme les producteurs européens se sont parfaitement accommodés, a posteriori, de la réforme de 1992 lorsque les aides fixes à l'hectare se sont révélé apporter une compensation supérieure à la baisse de revenu induite par la baisse des prix.) Mais, comme l'imagination politique $\mathrm{n}^{\prime}$ a pas de limite, rien $\mathrm{n}^{\prime}$ interdit d'envisager, les années où les prix remontent, une contribution exceptionnelle à l'assurance-revenu, calée sur les surfaces historiques, qui permette opportunément de retourner dans les caisses de l'État, cette rente indue.

Au-delà des astuces techniques qui permettent de métamorphoser des aides couplées en aides veaux dispositifs d'assurance des producteurs contre le risque. Initialement permise par le soutien des prix, l'assurance est ensuite passée par des paiements compensatoires directs. En même temps que l'on fige ces paiements directs dans des aides découplées, on introduit aujourd'hui des dispositifs d'assurance-revenu, au financement desquels les pouvoirs publics contribuent toujours activement... On prétend donc rétablir le signal des prix, mais, paradoxalement, lorsque ce signal commence à s'allumer, on le déconnecte. Lorsque les prix baissent suffisamment pour dissuader les agriculteurs de produire, on brouille le message en leur signifiant qu'on leur garantira un minimum de revenu quoi qu'il arrive. Autant dire que des aides, même parfaitement découplées, combinées à un système d'assurance revenu efficace, jouent à peu près comme un soutien des prix. On retrouve là une situation que les économistes ruraux les plus perspicaces ont déjà analysée depuis longtemps: la difficulté d'une régulation de la production par les prix sur des marchés agricoles instables et chaotiques, et l'utilité d'outils d'ajustement de l'offre tel que les quotas [10].

On en revient toujours au même constat : les marchés agricoles livrés à eux-mêmes ne permettent pas d'assurer un développement agricole durable et le découplage n'est pas une solution suffisante pour remédier à cet état de fait. Même découplées, les aides directes, aux États-Unis comme en Europe, restent pour l'essentiel des aides ciblées sur le soutien à l'activité marchande. Ceci a même conduit certains analystes à considérer le découplage comme une forme de dumping international au même titre que les restitutions à l'exportation [11]. Cette dernière thèse est sérieusement consolidée par les récentes décisions en appel de I'organe de règlement des différends de I'OMC, concernant la plainte déposée contre le système de soutien à la production de coton aux États-Unis, puisque le jugement considère que la séparation des soutiens entre soutiens internes et soutiens aux exportations est arbitraire, et que les soutiens internes peuvent jouer comme des soutiens à l'export. Ce faisant, I'OMC ouvre une véritable boîte de Pandore, car le dispositif américain de soutien au coton joue de la même manière pour le blé, le mais, le soja et l'arachide...Et rien n'interdit de penser que la logique, poussée à son terme, ne conduise à remettre en cause aussi les nouvelles aides découplées de l'Europe, comme soutien indirect à l'exportation. Dans la logique libérale de l'OMC, le devenir des aides découplées de la boîte verte n'est peut-être pas aussi assuré qu'on l'imagine, et la position de négociation de l'Europe n'est peut-être pas aussi forte que I'ont laissé entendre les commissaires Fischler et Lamy. 


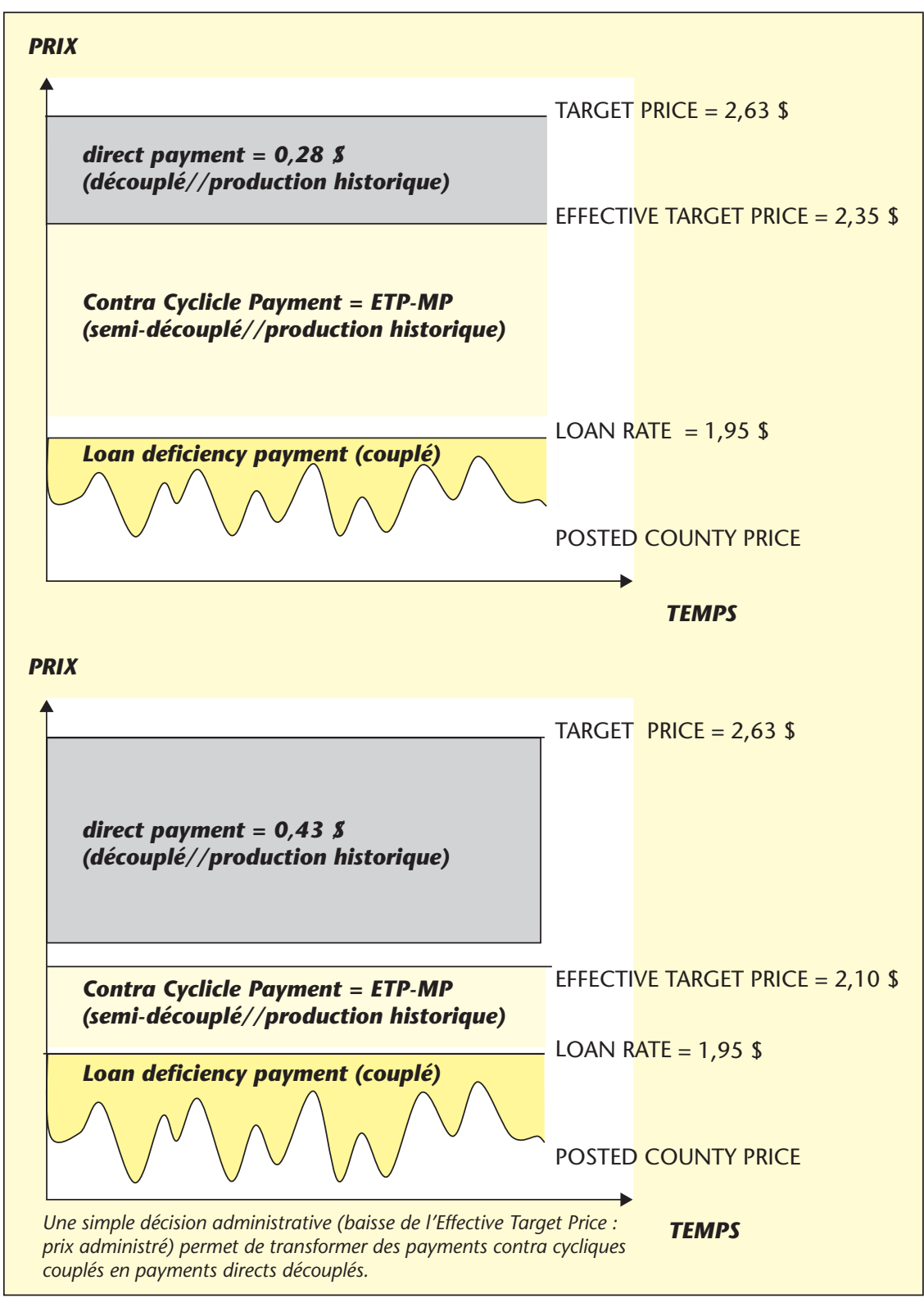

Figure 2. Transformation de soutiens semi-couplés en soutiens découplés.

\section{Durabilité sociale et environnementale : quelques contradictions de la PAC}

La nouvelle PAC s'assigne des objectifs ambitieux puisqu'il s'agit simultanément d'améliorer la compétitivité internationale de l'agriculture européenne, tout en assurant un développement agricole durable.

Au-delà des questions que pose l'affirmation de la « compétitivité » de l'agriculture européenne, dans le champ de la durabilité économique, quel diagnostic porter sur l'impact de la nouvelle PAC en matière de durabilité sociale et environnementale du développement agricole?
Il est, à vrai dire, difficile de se prononcer sur la dimension sociale du développement agricole qu'entend induire la PAC, car aucun objectif n'est explicité en la matière, si ce n'est peutêtre celui d'une parité de revenu avec les autres catégories socioprofessionnelles, objectif qui, dans un pays comme la France, est loin d'être atteint pour une part importante des exploitations (voir la $1^{\text {re }}$ partie : $45 \%$ des exploitations professionnelles françaises dégagent un résultat par actif familial - RCAI/UTAF - négatif ou inférieur au SMIC). La parité de revenu est, il est vrai, difficile à appréhender et dépend de l'échelle d'observation. Si l'on considère le résultat net de la branche par actif, il est proche du revenu moyen par actif dans l'ensemble de l'économie, avec des périodes alternées de décrochage et de rattrapage [12] tandis que les inégalités de revenus paraissent du même ordre de grandeur, dans la branche agricole, qu'au sein de la population active globale.

En matière d'emploi, d'effectif et de répartition des exploitations sur le territoire, la PAC ne $s^{\prime}$ assigne pas d'objectifs explicites précis. Les prévisions tablent sur une poursuite de l'exode agricole à un rythme de l'ordre de $3 \%$ par an, ce qui laisse à penser que la poursuite du mouvement de restructuration à l'œuvre sur la longue période reste un objectif social implicite [13]. En matière d'emploi, en effet, il semble que ce soit aux autres secteurs d'activité qu'il appartienne, comme par le passé, de résorber l'exode agricole, ce qui explique qu'en matière de développement rural, la politique de l'Union européenne s'appuie prioritairement sur l'essor des secteurs non agricoles, plutôt que sur un développement de la valeur ajoutée et un maintien de I'emploi dans l'agriculture. On peut donc raisonnablement estimer que le maintien de la population active agricole sur le territoire ne constitue pas un critère déterminant de durabilité de l'activité agricole, dans le dispositif actuel de la PAC. De fait c'est essentiellement en matière d'environnement que s'expriment les préoccupations de la PAC, au point qu'on peut considérer qu'il y a, dans l'esprit du législateur européen, une quasisuperposition de l'objectif de «durabilité » du développement agricole et de "soutenabilité environnementale » de ce développement.

En ce domaine, les indicateurs synthétiques d'impact, nous l'avons exposé, manquent encore cruellement. L'impact de la politique sur la durabilité environnementale du développement agricole ne peut donc être analysé que de manière très partielle, à partir d'exemples ou d'évaluations détournées. Dans un travail consacré à la "multifonctionnalité », nous avons cherché à évaluer les montants budgétaires consacrés à des objectifs environnementaux et de soutien à des formes d'agriculture durables [14]. II ressort de nos estimations que, même appréhendés de manière extensive, ces soutiens à la multifonctionnalité ne représentent en France qu'une part relativement modeste $-15 \%$ environ - des soutiens directs totaux consacrés à l'activité productive agricole (1,6 milliards d'euros sur un total de 10,7 milliards d'euros en 2001). Mais ces estimations quantitatives restent limitées. L'introduction dans la dernière réforme de la PAC de critères d'écoconditionnalité pour l'attribution des aides découplées constitue par exemple une avancée qualitative importante que nos comparaisons budgétaires (faites avant 2003) ignorent. Présent depuis longtemps dans les discours, I'objectif de durabilité environnementale s'inscrit donc progressivement dans 
les dispositifs de politique agricole, par une lente incrémentation qui a débuté en 1992 avec la mise en place des mesures d'accompagnement agro-environnementales, et qui s'est pour suivi en 2003 avec la mise en place de l'écoconditionnalité.

Toutefois, un certain nombre d'illustrations permettent d'évaluer le chemin qu'il reste à parcourir. L'exemple le plus immédiat a longtemps été le déséquilibre entre le soutien aux cultures intensives de maïs pour I'alimentation du bétail, réputées particulièrement agressives pour l'environnement, et le soutien aux cultures herbagères, avec une prime à l'hectare de maïs irrigué de l'ordre de $500 €$, dix fois plus élevée que la prime à l'herbe de $50 €$ accordée aux surfaces herbagères extensives... D'une manière générale, il est aisé de montrer que, même après 1992, la PAC a privilégié les systèmes de production les plus intensifs (au nom de la compensation calculée sur une référence historique) par rapport aux systèmes plus extensifs, en dépit des efforts de correction entrepris (évolution du calcul des rendements de référence). II est facile de montrer, pour la même raison, qu'elle a privilégié les régions intensives riches, en dépit des mécanismes correcteurs de compensation des handicaps naturels [15].

Le découplage accru des aides, instauré par la nouvelle PAC, devrait permettre de remédier en partie à cet état de fait. En effet, le producteur de maïs pourra désormais conserver, en France, $75 \%$ de sa prime désormais découplée, s'il convertit ses surfaces de mais en luzerne ou autres cultures herbagères, ce qui peut avoir un rôle incitatif important. On relèvera toutefois I'iniquité de traitement à laquelle conduit cette nouvelle disposition, puisque le producteur en système herbager sans maïs, qui a fait l'effort d'innover dans un système de production plus durable, se retrouvera sans aucune aide et déloyalement concurrencé, si bien que la réforme induit en fait une prime aux anciens pollueurs et une pénalité aux agriculteurs innovants dans l'agriculture durable.

Et rien ne prouve en outre que la réforme suffise partout à dissuader le développement des systèmes de production intensifs agressifs pour I'environnement. En production laitière, par exemple, les expertises dont on peut disposer [16] montrent que la réforme relancera la concentration géographique des productions et que, faute de possibilité d'agrandissement à court terme, le desserrement de la contrainte des quotas, conjugué à la baisse du prix, conduira dans les régions déjà intensives à un nouvel accroissement de la productivité physique des animaux et des chargements donc à un nouvel accroissement de la consommation $d^{\prime}$ aliment concentré et à un nouvel accroisse- ment de la pression environnementale liée à la résorption des déjections.

D'une manière générale, on peut craindre que le découplage, surtout lorsqu'il est intégral, ne démobilise prioritairement la production dans les régions extensives, accroissant encore la désertification et la fermeture de ces espaces, tandis qu'il aura un effet dissuasif marginal voire nul dans les régions intensives. Tant que les produits à l'hectare hors aides couvrent les coûts de production - ce qui continuera à être le cas dans les régions les plus intensives - la poursuite de l'intensification restera rentable. Dans le cas inverse, notamment dans les régions les plus pauvres, le passage à un système de culture minimum - le minimum requis pour toucher les droits à paiement unique peut devenir intéressant. Alors se concrétisera, au sens propre, la fonction de cantonnier de la nature. D'ailleurs, n'est-ce pas pour éviter des évolutions perverses de ce genre que la prime à la vache allaitante a été maintenue couplée en France, ainsi qu'une partie de la prime aux cultures (25\%) ? La question reste toutefois posée de savoir combien de temps pourra durer cette dérogation au découplage intégral, dans le processus de réforme engagé par la Commission.

En matière environnementale, il faut également rappeler l'impasse protéique dans laquelle se trouve l'Europe. Les légumineuses riches en protéines constituent, les agronomes le savent bien, une tête d'assolement particulièrement intéressante, économe en intrants, et très améliorante pour les sols. Or, I'Europe, depuis les accords du Dillon Round en 1962, bénéficie d'un système de prix défavorable aux oléoprotéagineux et aux légumineuses. Malgré un rééquilibrage des importations d'aliments du bétail après la réforme de 1992, qui a surtout concerné les céréales, elle reste massivement importatrice de protéines végétales pour I'alimentation animale. (11 millions de tonnes de tourteaux de soja importées en 1996, 17 millions de tonnes en 2002- UNCTAD 2003.) Bien plus, la réforme de 1999 a remis en cause les bénéfices des politiques antérieures de soutien spécifique à ce type de culture, en réduisant les primes dont elles pouvaient bénéficier (prime unique par hectare, alignée sur les surfaces en céréales) et la réforme de 2003 ne s'accompagne d'aucun réajustement en la matière. Cette fois encore, le primat du marché I'aura emporté sur la promotion d'assolements plus durables...

Que dire enfin des nouveaux droits à paiement unique? Ils constituent en quelque sorte un contre-statut du fermage. Avec le statut du fermage, renforcé par le décret de juillet 1965 portant création des prêts fonciers bonifiés à l'agriculture, le législateur s'était efforcé de limiter l'investissement stérile dans la capitali- sation foncière, pour permettre aux agriculteurs d'orienter prioritairement leur capacité de financement vers les investissements productifs. La capitalisation dans ce nouveau « fond d'entreprise » que constituent les nouveaux droits à prime constitue, à l'inverse, un détournement stérile de la capacité de financement au détriment des investissements productifs. Et quand nous parlons d'investissements productifs, il ne s'agit pas prioritairement d'investissements dans la productivité physique et l'augmentation de la production, mais bien d'investissements dans la productivité environnementale des systèmes de production...

\section{De la soutenabilité des politiques agricoles contemporaines}

La soutenabilité politique est une dimension qui n'est pas explicitement identifiée dans les définitions usuelles de la durabilité. On peut imaginer qu'elle constitue une composante de la durabilité sociale. Quoi qu'il en soit, dans une démocratie, une politique de soutien à un développement durable ne peut devenir efficace, que pour autant qu'elle agisse dans la durée, que pour autant qu'elle s'inscrive dans une échelle de valeur (équité, solidarité, mérite...) susceptible de faire consensus.

De ce point de vue, la nouvelle PAC paraît énormément fragile. Son financement jusqu'en 2013 n'est pas définitivement assuré et le nouveau règlement de développement rural pour la période 2006-2013, qui devait constituer le fer de lance de soutien à la durabilité dans la nouvelle politique, joue finalement le rôle de variable d'ajustement et ne dispose que d'un budget très réduit, pas du tout à la hauteur des enjeux de cohésion et d'équité qu'exige la nouvelle configuration de I'Union européenne, élargie à 25 États. En outre, les conditions de mise en œuvre de la nouvelle PAC sont extrêmement hétérogènes. Avec les multiples options de découplage à la carte, de possibilité de références individuelles ou régionales, de dispositifs nationaux spécifiques, de financements complémentaires, que reste-t-il des organisations communes de marché ? La solidarité financière risque alors d'être mise à mal, compte tenu de la réticence de certains États membres à alimenter le budget commun, compte tenu aussi de la part croissante des actions cofinancées par les États (deuxième pilier), compte tenu enfin du projet implicite de la Commission de renvoyer aux États la charge de la gestion du risque. N'est-ce pas finalement la renationalisation des politiques agricoles qui s'annonce? Comment dès lors les États ou les collectivités territoriales pourront-ils se saisir du développement agri- 
cole, comment pourront-ils agir pour la promotion d'un développement agricole durable ? En l'absence de toute solidarité fortement affirmée entre les États, les régions et les différentes catégories d'agriculteurs, la renationalisation, voire la régionalisation des politiques agricoles, ne portent-elles pas en germe les risques d'une concurrence accrue entre les pays les plus riches de l'Union et les pays les plus pauvres, entre les régions les plus riches et les régions les plus pauvres? Car l'une des caractéristiques de la politique agricole commune, que les économistes libéraux lui ont d'ailleurs souvent reprochée, c'est de n'avoir fait jouer que de manière très modérée la concurrence entre pays de I'Union. Globalement, tous les pays de I'Union ont développé leur agriculture et leur degré d'autosuffisance [17] et l'on peut considérer que cela a constitué un facteur important de réussite et de durée de la PAC [18]. On a, par exemple, souvent critiqué les restitutions importantes à l'exportation dont ont pu bénéficier les exportateurs français grâce au dispositif communautaire. Mais certains économistes allemands [19] expliquent bien que ces exportations sur les pays tiers, financées en grande partie grâce à la contribution budgétaire allemande, ont évité un ajustement par la baisse des prix qui, dans une logique de concurrence avivée entre les pays, aurait d'abord pénalisé la céréaliculture allemande, moins bien armée que la céréaliculture française ou anglaise, ou même danoise, pour supporter la baisse des prix.

En matière d'équité, toutefois, un certain nombre d'interrogations restent posées, bien que la question ait été soulevée dès 1992. En dépit des pétitions de principe avancées à chaque étape, les réformes successives sont restées extrêmement timides quant à la question de la répartition des soutiens publics entre les différentes catégories d'exploitation, entre les différentes régions, entre les différentes orientations de production. Certes, on peut, dans un pays comme la France, se réjouir de ce que les disparités de revenu interdépartementales se soient réduites de 1992 à 2002. Reste que l'écart de revenu net d'entreprise par actif non salarié entre le département le plus riche - la Marne - et le département le plus pauvre - la Dordogne - était encore dans une proportion de 1 à 20 en 2003 (moyenne 2001-20022003) alors qu'il était, il est vrai, dans une proportion de 1 à 30 en 1992 (moyenne 19911992-1993) [20]. Si I'on raisonne sur la moyenne arithmétique des dix départements les plus riches par rapport aux dix départements les plus pauvres, cet écart passe de 1 à 8,6 en 1992, à un rapport de 1 à 6,8 en 2002. Le découplage croissant des aides a donc bien permis une correction des disparités départementales de revenu dans l'agriculture fran- çaise, sans toutefois remettre en cause la hiérarchie très inégalitaire qui persiste encore en 2002. Dans la mesure où les aides directes constituent une part essentielle du revenu la hiérarchie des revenus apparaît aujourd'hui en grande partie liée aux modalités de répartition des aides. En l'absence de dispositifs de modulation des aides ou d'écrêtement, leur distribution proportionnelle aux surfaces et différenciée selon les orientations de production fige en grande partie les situations historiques acquises. La question d'une meilleure équité dans la répartition des aides, posée depuis 1992, reste aujourd'hui très actuelle, avec une acuité renforcée dans une Europe élargie à 25 États, qui connaît désormais des disparités régionales considérablement accrues, ce qui renforce l'interpellation quant à la soutenabilité politique des formes actuelles de répartition des soutiens...

Au-delà du débat sur l'équité des soutiens, la soutenabilité des politiques agricoles concerne aussi des enjeux moins médiatisés, mais d'une importance sociale peut-être plus essentielle encore. Pour illustrer cela, repartons d'un nouvel exemple qui nous vient des États-Unis. La dernière enquête nationale sur les travailleurs agricoles (mise en place en 1988 pour aider à la gestion de programmes sociaux) révèle que plus de la moitié des travailleurs employés dans I'industrie agroalimentaire serait constituée de travailleurs immigrés en situation irrégulière. "On estime aujourd'hui que les immigrés en situation irrégulière occupent près de $60 \%$ de la main-d'œuvre salariée agricole américaine. Cette source de main-d'œuvre est généralement payée au salaire minimum (variable selon les États et de I'ordre de 7 \$ par heure) sans contrepartie sociale de l'employeur. » [21].

Il y aurait certainement intérêt à évaluer ce que cela signifie comme soutien indirect à l'agriculture américaine mais I'OMC ne veut pas connaître ce type de distorsion de concurrence. Au plan qui nous intéresse, la question reste posée de la validité d'une telle politique, dans la perspective d'un développement agricole durable. Quel est le prix politique de la durabilité ? Et la question risque de ne pas concerner que les États-Unis. Le phénomène est connu aux États-Unis parce qu'il est étudié. $C^{\prime}$ est si vrai que le ministère du travail fédéral américain a, un moment, envisagé de résoudre le problème... en interrompant l'enquête. Mais qu'en est-il en Europe ? Difficile de le savoir faut d'enquête exhaustive, mais un certain nombre de faits divers dramatiques restent révélateurs d'un malaise certain concernant l'emploi massif de travailleurs clandestins dans l'agriculture européenne [22].

\section{Des règles internationales inadaptées}

Dans le contexte actuel, les politiques agricoles, en dépit de leurs ambitions affichées, paraissent encore bien peu avancées pour soutenir un développement durable de l'agriculture. Or, depuis 1994 ces politiques sont étroitement encadrées par les accords commerciaux internationaux, en tout premier lieu I'accord agricole de l'Uruguay Round, entré en vigueur en 1995 et dont la renégociation est aujourd'hui dans l'impasse. De fait, les principes économiques qui fondent ces politiques ont été établis dans le cadre de concertations multilatérales internationales, proposées par I'OCDE et codifiées par I'organisation mondiale du commerce.

Sans revenir sur le cadre théorique sous-jacent, qui justifie le découplage, le classement des aides dans les différentes boîtes, la référence aux cours mondiaux comme prix d'équilibre, le calcul des ESP et la classification des différentes formes de soutien, se pose la question de l'efficacité concrète de ces politiques. Après tout, peu importe qu'une politique soit fondée sur des principes erronés, pourvu qu'elle atteigne les objectifs recherchés. Or, en ce domaine, les résultats observés sont loin des bénéfices attendus.

Découplage ou pas, les soutiens à l'agriculture n'ont pas diminué. Le soutien aux producteurs (ESP) calculé par I'OCDE était en moyenne de $30 \%$ de la production pour l'ensemble des pays de l'OCDE en début de période (1995). ॥ est monté jusqu'à $35 \%$ en 1999, pour retrouver son niveau de $30 \%$ en 2002 [23]. Aux États-Unis, les soutiens budgétaires directs ont augmenté brutalement à la fin des années 90 , passant de 7 milliards de dollars en 1997 à 37 milliards en 2001 (USDA 2004). Ils sont stabilisés en Europe depuis 1992 autour de 40 milliards d'euros. Toutefois, compte tenu de la diminution de la population active agricole dans tous les pays développés, les soutiens par actif se sont considérément accrus aux ÉtatsUnis et ont continué d'augmenter en Europe. On peut certes se consoler, avec l'OCDE, en remarquant que I'on a transformé des mauvais soutiens en bons soutiens, mais la consolation ne vaut que pour autant que vaille la classification entre bons et mauvais soutiens (question qui a précédemment été discutée).

Le découplage et l'ouverture des frontières devaient, quant à eux, conduire à une meilleure flexibilité de l'ajustement de l'offre et de la demande et à une meilleure régularisation des cours des transactions... Dans les faits, les cours internationaux n'ont rien perdu de leur volatilité, avec une succession de flambées (1996) et d'effondrements (1998-1999). On peut même considérer qu'en substituant des 
aides découplées, sans contraintes, à des aides couplées, mais assorties de contraintes de mâ̂trise des volumes (quotas, jachères obligatoires), on a détruit les quelques instruments de régulation des échanges internationaux qui subsistaient. Rappelons qu'en 1983, avant le découplage, les Etats-Unis ont gelé jusqu'à 30 millions d'hectares de surfaces de culture (équivalent à la SAU française et à la sole céréalière européenne). Nul doute que cette intervention pour redresser le marché domestique du premier exportateur mondial de céréales n'ait eu un effet indirect bénéfique sur l'échange international. On comprend, face à la concurrence de nouveaux exportateurs, que les États-Unis n'aient pas souhaité continuer à assurer seul, au profit des autres, cet effort d'assainissement. Mais, faute d'élaboration d'un nouveau consensus entre exportateurs pour partager l'effort de maîtrise de l'offre sur le «marché mondial », le consensus réalisé à l'OMC sur le découplage apparaît comme un consensus a minima, qui consiste à décharger les pays exportateurs de toute responsabilité dans la régulation des échanges internationaux. Le découplage est donc en quelque sorte la consécration de la renationalisation des politiques de gestion du risque, à l'échelle internationale des grandes régions, comme au sein de l'Union européenne.

Les règles internationales $d^{\prime}$ organisation $d u$ commerce ignorent aussi, nous l'avons souligné, les questions que soulève l'endettement des pays en développement et les fluctuations de parités monétaires. L'amnésie de l'Europe, en ce domaine est étonnante. N'est-ce pas en Europe, précisément, que l'on a inventé les montants compensatoires monétaires, pour enrayer les effets de distorsion de concurrence que risquaient de provoquer, à l'époque, les différentiels de change entre les monnaies des différents pays de I'Union? Pourquoi, ce qui est apparu bon pour l'Europe dans les années 70 a-t-il été complètement ignoré dans les négociations commerciales de I'Uruguay Round? Comment expliquer que I'on puisse être aussi ferme sur le démantèlement des protections douanières, parfaitement transparentes, et aussi laxiste quant aux protections monétaires, infiniment plus insidieuses? Comment espérer orienter les agriculteurs européens vers un développement durable, dans un univers où les signaux économiques sont délibérément faussés ? Comment, de la même manière, espérer une moralisation des règles du commerce international quand le poids de l'endettement accule les pays les plus touchés à l'exportation à n'importe quel prix (dumping fiscal, social, environnemental), et ce, le plus souvent, avec le soutien du FMl et de la Banque mondiale? [24]. Loin de soutenir la durabilité, les règles actuelles de l'échange international, en assu- rant le primat de la concurrence sur les autres droits, sape tout effort pour construire des règles nouvelles susceptibles d'élargir la définition du développement, pour un développement économique et agricole plus durable. Nous touchons ici une question politique essentielle. La durabilité $n^{\prime}$ est pas une construction linéaire additive: une bonne durabilité économique plus un peu de durabilité environnementale, avec un zest de durabilité sociale... La durabilité, pour devenir effective, suppose au contraire la capacité d'intégrer et d'arbitrer, dans une approche globale du développement, les différentes dimensions économiques, sociales et environnementales d'une croissance maîtrisée, tournée vers la satisfaction des besoins diversifiés, marchands, culturels, environnementaux des populations du globe. Les formes modernes de l'État qui se sont consolidées au XXe siècle ont incarné, au niveau national, cette capacité collective d'arbitrage du développement entre les différentes aspirations sociétales (parmi lesquelles il est vrai, les aspirations environnementales sont longtemps restées à la mesure des problèmes posés, c'est-àdire relativement modestes).

L'internationalisation des mouvements de marchandises et de capitaux a conduit à des transferts de compétence multiples des États vers des instances régionales et internationales nouvelles ou renforcées (Union européenne, OMC, $\mathrm{ONU}$ ) par le truchement notamment de multiples accords internationaux, dans des domaines très variés, économiques, commerciaux, militaires, culturels, environnementaux, etc. Un nouveau droit international se construit donc, à partir de ces dispositifs partiels, éclatés, sans qu'il existe $d^{\prime}$ instance internationale susceptible d'en unifier les principes ou, tout au moins, d'en arbitrer les contradictions, comme ont longtemps été en mesure de le faire les États-Nations. Nous sommes donc à une période charnière où les États-Nations se sont défaits d'un certain nombre de prérogatives, sans qu'aient émergé des formes de gouvernance supranationales nouvelles, qui disposent de la légitimité démocratique nécessaire pour assumer les fonctions d'arbitrage indispensable à un développement plus durable, politiquement soutenable. Faut-il alors poursuivre la fuite en avant en partant du principe que la nécessité fera force de loi et que, lorsque les problèmes se poseront de manière suffisamment criante, il faudra bien les résoudre? Faut-il au contraire consolider les formes régionales de régulation en émergence avant d'aller plus loin? Et, pour construire ces formes nouvelles de régulation régionales, jusqu'où la règle de la subsidiarité peut-elle être opérante? Peut-on arbitrer les règles de la concurrence à Genève, les règles de la politique agricole à Bruxelles, les règles de la solidarité sociale à
Paris, et les politiques du développement économique dans les chefs-lieux de région? L'une des difficultés du débat que la France vient de connaître concernant la construction européenne ne vient-elle pas de la distorsion d'un dispositif qui prétend arbitrer la concurrence à l'échelle européenne, mais laisser l'arbitrage de la répartition au niveau des États ? Si la concurrence augmente les richesses créées en Europe, où doivent se situer les instances qui permettront d'arbitrer une répartition équitable de ces richesses nouvelles entre les Européens?

Compte tenu de tout ce qui vient d'être dit, les institutions internationales apparaissent pour l'instant particulièrement mal armées pour induire des politiques de soutien efficaces à un développement agricole durable, en raison des rapports de pouvoir très inégaux dans lesquels elles se trouvent, en raison également de la spécialisation de leurs missions. Parmi les multiples institutions internationales, trois jouissent, de fait, d'un pouvoir infiniment plus important que toutes les autres: le FMI, la Banque mondiale et I'OMC. Mais I'une s'occupe des questions monétaires, la deuxième de financement, la troisième de commerce, aucune de développement durable...

Concernant les politiques agricoles dans les pays développés, c'est désormais l'OMC qui joue un rôle moteur de définition, $d^{\prime}$ orientation et de sanction. C'est moins vrai dans les pays en développement, dans lesquels le FMI ne s'interdit pas de conseiller les pays endettés en matière de politique agricole et commerciale, mais c'est le plus souvent pour renforcer ou pour anticiper les préoccupations de libéralisation des échanges qui se négocient à l'OMC. Pour ces institutions dominantes, la meilleure politique de développement, c'est une politique de libéralisation commerciale, la meilleure politique agricole, c'est une politique d'insertion accélérée dans les échanges internationaux.

Pourtant, s'il est un secteur d'activité où il apparaît de manière criante que la libéralisation des échanges ne peut tenir lieu de politique de développement et encore moins de développement durable, c'est bien l'agriculture. Agriculture et alimentation sont étroitement liées, et le libre jeu de marchés, qui ne concerne par définition que les consommateurs solvables, ne peut prétendre répondre aux besoins alimentaires les plus pressants des populations les plus démunies et, en premier lieu, des populations rurales. Au risque de répétition, rappelons ici encore ce qu'est la réalité des agricultures du monde [25]. L'agriculture du monde, c'est $d^{\prime}$ 'abord une agriculture vivrière qui concerne quelque 1200 millions d'actifs agricoles, qui a peu évolué au cours du siècle, faute de capacité d'épargne et d'investissement. En regard, 
I'agriculture mécanisée des pays du Nord et des pays émergents reste le fait de quelque 30 millions d'actifs, qui ont eu la possibilité d'augmenter considérablement leur productivité de travail durant les cinquante dernières années. L'une, de loin la plus nombreuse, stagne tandis que l'autre, extraordinairement minoritaire, décolle. En résultent aujourd'hui des écarts de productivité de l'ordre de 1 à 1000 (10 quintaux par actif et par an chez les uns, 10000 quintaux par actif et par an chez les autres). Dans ces conditions, les écarts de productivité physique sont tels qu'il devient parfaitement absurde de confronter sur un même marché des catégories aussi éloignées de producteurs. De puissants mécanismes correcteurs s'imposent impérativement, car le marché concerne tous les producteurs, y compris les agricultures vivrières. En effet, même si l'agriculture vivrière consomme l'essentiel de sa production, elle a besoin d'un minimum de recettes monétaires pour assurer les dépenses élémentaires indispensables à la reproduction de son activité. Exposées sans protection à la concurrence internationale et à la baisse des prix, ces agricultures vivrières se voient confisquer leur maigre recette monétaire, ce qui sape ainsi les bases de reproduction des systèmes vivriers. Le jeu de la compétitivité internationale conduit alors à transformer des agriculteurs pauvres, qui subviennent tant bien que mal à leurs besoins, en inactifs totalement dépendants qui, chassés des campagnes, grossissent les rangs des populations marginalisées des périphéries urbaines, à la recherche d'hypothétiques activités de survie. La libéralisation des échanges alimentaires se révèle ainsi une redoutable machine planétaire pour entretenir la pauvreté et l'exclusion. Le poids numérique déterminant des paysans pauvres dans l'agriculture mondiale, I'enjeu primordial d'une sécurité alimentaire minimale pour la moitié des habitants de la planète devraient suffire à convaincre qu'il y a bien une exception agricole et qu'il est absurde de prétendre gérer les échanges alimentaires internationaux avec les mêmes règles que les biens manufacturés. La première condition d'un développement agricole durable, c'est donc, de toute urgence, la protection des agricultures les plus pauvres, qui restent les plus indispensables à la résolution de la question alimentaire mondiale. II apparaît, a contrario, parfaitement illusoire de prétendre alimenter ces populations les plus démunies, donc les moins solvables, avec les excédents des pays riches, mêmes bradés à bas prix sur les marchés internationaux.

\section{Quelques points clefs pour conclure}

Le développement durable est une préoccupation récente qui a envahi l'espace politique.
Pour l'économiste, le développement durable reste une construction inachevée qui, pour devenir opératoire, doit pouvoir s'appuyer sur des indicateurs synthétiques reconnus, afin d'objectiver, à partir d'observations mesurables, les intentions méritoires des décideurs politiques.

En matière d'agriculture, le défi d'un développement durable paraît d'une ampleur particulière en raison du rôle spécifique que joue la question alimentaire dans la satisfaction de besoins essentiels, et en raison du poids spécifique des agricultures paysannes pauvres dans l'agriculture mondiale. Répondre à ces défis implique de refonder les politiques agricoles sur des bases économiques qui puissent $s$ 'affranchir des représentations mythiques de marchés concurrentiels parfaitement efficients, pour fonder l'action sur une analyse rigoureuse de la diversité des structures de production, et du fonctionnement des marchés réels.

Cela suppose, en matière d'organisation de marchés agricoles, de refonder les politiques sur le principe de la vérité des prix. Concevoir I'organisation des marchés et des échanges à partir d'un « prix mondial » qui ne couvre pas les coûts de la production marchande des agricultures les plus modernes du monde est une absurdité. Qui, en effet, peut oser prétendre que l'agriculture européenne, l'agriculture américaine, l'agriculture japonaise ne sont pas nécessaires à l'approvisionnement alimentaire de la planète ? Qui peut prétendre que le Brésil, I'Australie, I'Argentine et la Nouvelle-Zélande sont capables d'assurer seuls la sécurité alimentaire du monde ? Le Brésil reste en ce domaine un exemple à méditer: la frange la plus moderne de son agriculture qui se prépare à pousser ses exportations vers les pays les plus solvables, côtoie une masse importante de paysans pauvres ou sans terre, tandis que les énormes besoins du marché domestique restent insatisfaits, faute de consolidation d'une demande solvable et d'une répartition équitable des moyens de production agricole et alimentaire...

Encore n'évoquons-nous pour l'instant que la simple durabilité économique de la production marchande... La vérité des prix, pour un développement agricole durable, nécessite une prise en compte des coûts de production totaux réels: coûts d'amortissement et de renouvellement des équipements achetés, mais aussi coûts de renouvellement de l'écosystème cultivé, qu'ils soient marchands et directement identifiables ou non marchands et relevant d'indicateurs spécifiques. À l'inverse, la référence au seul «prix mondial» conduit aujourd'hui dans les pays développés, mais encore plus dans les pays en développement, à un dumping social et environnemental, qui favorise des modèles de « développement » de très court terme, dont la logique se situe aux antipodes d'un développement durable... De fait, quand I'OCDE, I'OMC ou la Banque mondiale estiment les augmentations de bien-être liées à l'intensification accélérée des échanges internationaux, il n'y a aucune prise en compte, dans ces calculs, des coûts environnementaux indirects (épuisement des réserves énergétiques, dégagement de gaz à effet de serre, etc.) liés à l'intensification des échanges, aucune anticipation même de l'augmentation inéluctable des coûts de transport (le coût du fret international en vrac transatlantique est aujourd'hui de l'ordre de $50 \$$ la tonne, pour un prix du baril de pétrole de $50 \$$ et un prix de la matière première comme le maïs de l'ordre de $100 \$ /$ tonne. Le coût du fret représente donc déjà la moitié du coût de la marchandise. Qu'en sera-t-il lorsque le prix du baril atteindra les $380 \$$, comme le prévoit Patrick Arthus, économiste en chef de la Caisse des dépôts? [26].

La maîtrise des prix constitue donc un enjeu déterminant pour la promotion d'un développement agricole durable. En même temps, nous avons vu que la gestion de la durabilité supposait une approche multidimensionnelle de l'activité de production, dans ses dimensions économique, sociale et environnementale, donc une forme d'organisation institutionnelle qui permette un arbitrage économique efficace et politiquement acceptable, entre ces objectifs multiples. En matière agricole, compte tenu des hétérogénéités considérables qui existent entre les agricultures du monde, nous pensons avoir montré que la fuite en avant irraisonnée dans la globalisation à tout prix risquait de conduire dans une impasse. Entre l'État national, souvent impuissant à maîtriser les effets de l'internationalisation des échanges, et la fuite en avant vers une hypothétique gouvernance mondiale, il y a place pour une structuration politique et institutionnelle du développement agricole à une échelle régionale appropriée, entre pays de niveaux de développement comparables, au sein desquels la question de la durabilité se pose de manière convergente. Or, une telle organisation institutionnelle ne naît pas spontanément de la libéralisation des échanges, mais d'un processus de construction politique. II convient alors de pousser l'analyse jusqu'à son terme. Pour qu'une organisation économique et politique régionale puisse s'affirmer et faire jouer ensemble les leviers de l'efficacité économique, de la solidarité sociale et de la responsabilité environnementale, elle doit pouvoir maîtriser ses prix et réguler ses échanges avec le reste du monde. La protection aux frontières reste donc nécessaire, pour promouvoir un développement agricole durable, organisé dans des zones socioéconomiques intégrées. II reste une 
nécessité pour éviter que des pans entiers des économies régionales, en premier lieu les productions vivrières, ne soient détruits par des prix internationaux abusivement bas, notamment lorsque les écarts de productivité entre I'intérieur et l'extérieur sont trop grands.

Dans cette logique de structuration régionale, l'expérience européenne reste utile à méditer. L'intégration agricole n'a été possible que par une protection du marché domestique (préférence communautaire), ce qui n'a d'ailleurs pas empêché I'Union européenne d'augmenter régulièrement ses importations et de devenir la première zone importatrice du monde de produits agricoles et alimentaires. L'intégration économique a été rendue possible par une réelle solidarité financière. Et si l'intégration européenne a été possible, c'est aussi parce qu'elle concernait des pays voisins, de niveau de développement économique comparable, et parce qu'ont été mis en place les instruments de solidarité nécessaires au maintien de la cohésion. Construire l'Europe à six, puis à douze, a été un pari réussi. Construire une Europe à vingt-cinq ou à vingt-sept, marquée par des disparités économiques considérablement accrues, est un pari beaucoup plus difficile, qui ne peut être gagné que sous condition d'un renforcement considérable de l'effort de solidarité et de cohésion économique, donc un renforcement des contributions budgétaires et de la protection par rapport au reste du monde. De ce point de vue, les évolutions actuelles de la politique agricole commune vont à l'encontre de ces objectifs, puisqu'elles proposent une dilution de la préférence communautaire dans la globalisation des échanges internationaux et une dilution de la solidarité par la renationalisation des politiques agricoles. Certes, les modalités de gestion des organisations communes de marché doivent être profondément révisées, pour prendre en compte les exigences d'un développement agricole durable en interne (répondre aux nouvelles demandes sociales qui s'expriment) et à l'international (respecter les exigences d'un développement durable dans les autres parties du monde). Mais la refondation d'une politique pour un développement agricole durable ne parait pas pouvoir se faire en oubliant les principes de base de la construction agricole commune, principes de la préférence et de la solidarité communautaire, principes d'équité interrégionale et de maîtrise des prix.

\section{RÉFÉRENCES}

1. RIST G. Le développement. Histoire d'une croyance occidentale. Paris : Presses de Sciences Po, 2001.

2. THIEBAUT L. 2005.

3. CHATELLIER V. 2005.

4. KROLL JC. Le volet agricole de l'agenda 2000 : un argumentaire économique controversable. Compte rendu de l'Académie d'Agriculture de France 1999 ; 85(3), Séance du 24 mars 1999.

5. MANDEL. DEBREU. In : Guerrien $\mathrm{H}$, ed. Dictionnaire d'analyse économique. Paris : La Découverte, 1996.

6. MILLET D, TOUSSAINT E. 50 questions, 50 réponses sur la dette, le FMI et la banque mondiale. Belgique : Edition Sylepse, 2002.

7. DEBAR JC. Les politiques de soutien à I'agriculture en Europe et aux États-Unis à travers les chiffres. Paris : Colloque SFER, 9-10 octobre 2002.

8. DOUMENC H, MENU F, TREGUER D. Evaluation des coûts de production en céréaliculture, comparaison des méthodes et des résultats en France et aux États-Unis. ENGREF-ONIC, Rapport TGE, mars 2003.

9. EDWIN YOUNG C. Developpement in US Agricultural Policy. Washington: Economics Research Service, USDA, 2004.

10. BOUSSARD JM. Faut-il encore des Politiques Agricoles?. Paris : DEMETER Armand Colin, 2002.

11. BERTHELOT J. L'agriculture, talon d'Achille de la Mondialisation. Paris : L'Harmattan, 2001.

12. INSEE. AGRESTE 2004. Les comptes de l'agriculture en 2003 - Rapport présenté à la Commission de comptes de l'agriculture de la Nation. Collection Synthèse $n^{\circ} 77$.

13. COMMISSION DES COMMUNAUTES EUROPEENNES. Communication de la Commission au Conseil et au Parlement Européen Révision à mi-parcours de la Politique Agricole Commune. COM 2002 ; 394. Bruxelles, 10 juillet 2002.
14. BAZIN G, KROLL JC. La multifonctionnalité dans la Politique Agricole Commune : projet ou alibi ? Session SFER, Paris, 22 mars 2002.

15. BAZIN. 1999

16. INSTITUT DE L'ELEVAGE. Réforme de la PAC et production laitière : perspectives d'évolution à I'horizon 2010-2012. Dossier Economie de l'Elevage $2004 ; 340$.

17. M. GRENIER ET T POUCH. 2001.

18. L. BOURGEOIS. C. SERVOLIN 2002.

19. AHRENS H. LIPPERT C. Politique agricole comparée de l'Allemagne et de la France. Economie Rurale 2002 ; mars-juin.

20. CARAES J. Départements français, une réduction des disparités de revenus. Chambre d'Agriculture $2005 ; 942$.

21. MINISTÈRE DE L'ECONOMIE ET DES FINANCES. FLASH AGRI-ZONE ALENA. USA: brouillard sur la main-d'œuvre clandestine. Ministère de $\mathrm{I}^{\prime}$ Economie et des Finances - $\mathrm{n}^{\circ}$ 43/mai 2005.

22. FORUM CIVIQUE EUROPÉEN. El Ejido, terre de non droit. Bâle/Limans : Editions Golias, FCECEDRI, 2000.

23. OCDE. Politiques agricoles dans I'OCDE, Panorama - Principales conclusions. Paris: OCDE, 2004.

24. STIGLITZ J. La grande désillusion. Paris : Fayard, 2002.

25. MAZOYER M, ROUDART L. Histoire des agricultures du monde. Paris : Le Seuil, 1997.

26. BOURGEOIS L. Le barril à 380 dollars! APCA Conjoncture agricole. Synthèse mensuelle $n^{\circ}$ 240, mai 2005.

\section{Compléments de lecture}

CNIEL. L'économie laitière en chiffres. Paris : Centre Interprofessionnel de documentation et d'information Laitière, 2003.

NUCED C. Atlas des produits de base Fond commun pour les produits de base, New-York et Genève. 2004 ; (2004).

THIEBAUT L. Glossaire d'environnement : économie, politiques, évaluation. Version provisoire, ENESAD, 2003 ; (520 p). 